\title{
La luminescence stimulée optiquement : Applications à la cartographie de dose et à la mesure de dose en environnement spatial
}

\author{
J. FESQUET ${ }^{1}$, D. BENOIT ${ }^{1}$, J.-R. VAILLÉ ${ }^{1}$, P. GARCIA ${ }^{1}$, H. PREVOST ${ }^{1}$, \\ J. GASIOT ${ }^{1}$, L. DUSSEAU ${ }^{1}$
}

(Manuscrit reçu le 6 novembre 2006, accepté le 28 février 2007)

RÉSUMÉ Nous dressons un état de l'art sur la dosimétrie des rayonnements par luminescence stimulée optiquement (OSL), développée à l'Institut d'Électronique du Sud (IES). Les propriétés spectrales de notre matériau permettent une détection de la lumière sans utilisation de filtre optique. Il présente une grande dynamique (supérieure à 7 ordres de grandeur), une sensibilité inférieure à $10 \mu \mathrm{Gy}$ et une réponse uniquement fonction de la dose absorbée. Des travaux réalisés au CERN ont montré une limite de saturation de l'ordre de 300 Gy. Deux types de dosimètres ont été développés : (1) un capteur intégré destiné à la réalisation de mesures de dose en ligne ; (2) des films OSL qui permettent la réalisation de contrôles géométriques des faisceaux d'irradiation (protons, électrons, alpha, photons). Nous étudions actuellement l'impact du dopage au bore sur notre matériau OSL dans le but de réaliser des mesures dosimétriques en champ mixte neutron - gamma. En parallèle, afin d'améliorer la reproductibilité et l'homogénéité des dépôts OSL, nous développons une méthodologie par couche mince.

ABSTRACT Optically stimulated luminescence: applications to dose-mapping and space dose measurement.

The Institut d'Électronique du Sud (IES) has developed a dosimetry technique based on optically stimulated luminescence (OSL). The state of the art is presented here. The OSL material's spectral properties allow a light detection with no additional filtration. This material exhibits a large dynamic range ( 7 orders of magnitude), a sensibility much of $10 \mu \mathrm{Gy}$ and a response which is strictly a function of the absorbed dose. Characterization carried out at CERN yielded a saturation limit around 300 Gy. Two different types of dosimeters have been developed: (1) an integrated sensor devoted to the on line radiation monitoring; (2) OSL films making possible the control of the geometry during irradiation with proton, electron, alpha, and photon beams. Currently, the effect of boron doping on OSL material is studied in order to enhance the performances in mixed field neutron - gamma. In parallel, we develop a new deposition technique based on thin layer in order to improve reproducibility and the homogeneity of the OSL films.

Keywords: optically stimulated luminescence (osl) / dosimetry / dose-mapping / sensors

${ }^{1}$ Institut d'électronique du sud (IES), UMR 5214 UM2-CNRS, université Montpellier 2, C.c. 083, Place Eugène Bataillon, 34095 Montpellier Cedex 5, France. 


\section{Introduction}

Depuis les années 1980, l'équipe « Électronique et Rayonnements » de l'IES s'est spécialisée dans l'étude des phénomènes de piégeage et de relaxation dans les isolants. La composante «Dosimétrie » est à l'origine de nouveaux capteurs et systèmes pour la métrologie et l'imagerie des rayonnements ionisants.

La mise au point en 1981 (Gasiot et al., 1981) d'un banc de lecture de dosimètres thermoluminescents (TL) par chauffage laser a permis de réduire les temps de lecture d'un facteur mille par rapport aux techniques conventionnelles. Dans ce système, le dosimètre TL est obtenu en déposant par sérigraphie une couche de matériau TL $\left(\mathrm{CaSO}_{4}\right.$ ou $\left.\mathrm{Al}_{2} \mathrm{O}_{3}\right)$ sur un film mince de $20 \times 20 \mathrm{~cm}^{2}$ en polyimide kapton. La stimulation de la surface du film, point par point, est obtenue à partir d'un laser $\mathrm{CO}_{2}$ de longueur d'onde $10,6 \mu \mathrm{m}$. Il est ainsi possible de lire des plaques thermoluminescentes avec une résolution inférieure au millimètre, la taille minimale des pixels étant limitée par la diffusion thermique au niveau du point chauffé par le laser. Toutefois, en dépit de temps de lecture pénalisants (environ 30 minutes), d'un lecteur lourd et d'utilisation très délicate, ces systèmes ont permis de démontrer l'intérêt de la cartographie de dose pour la caractérisation de faisceaux de particules et la mesure 2D dans des géométries complexes (Albert et al., 1997 ; Setzkorn et al., 1997, 1996 ; Beteille et al., 1996 ; Prévost et al., 1995 ; Setzkorn, 1995 ; Vignolo, 1994 ; Sevière, 1993).

En 1982, les travaux de Jean Gasiot sur les phénomènes de luminescence par stimulation optique (OSL, pour Optically Stimulated Luminescence) (Gasiot et al., 1982) ont mis en évidence l'intérêt de la stimulation optique par rapport à la stimulation thermique pour la cartographie de dose. Il a en effet expérimentalement démontré qu'il était possible au moyen d'un laser YAG déclenché de dépiéger en quelques nanosecondes un matériau OSL préalablement irradié. L'avantage de la stimulation optique sur la TL pour la cartographie de dose est donc double puisqu'elle autorise des temps de lectures beaucoup plus courts et que la résolution n'est pratiquement limitée que par la longueur d'onde de la lumière de stimulation (typiquement le micron), puisqu' on s'affranchit des effets thermiques.

Au début des années 1990, le laboratoire a développé une famille de dosimètres OSL utilisant des sulfures alcalino-terreux, qui ont une sensibilité suffisante pour les applications en dosimétrie (Missous, 1992 ; Loup, 1991). Ce sont les caractéristiques de ces matériaux qui ont été exploitées pour des applications dans les domaines de la cartographie de dose OSL mais aussi pour la dosimétrie en milieu hostile, embarquée sur les satellites (Idri, 2004 ; Vaillé, 2003 ; Plattard, 2001 ; Polge, 2001 ; Ranchoux, 2001 ; Albert, 1997 ; Beteille, 1995 ; Dusseau, 1995 ; Dusseau et al., 1995). 
Depuis la découverte des ceintures de radiations par Van Allen en 1958, les acteurs de la conquête spatiale ont été confrontés à un environnement radiatif hostile. Le problème se pose autant pour les équipages des missions habitées que pour les systèmes embarqués. Les équipements électroniques, bien que pouvant tolérer des niveaux de doses bien plus élevés qu'un organisme vivant, sont beaucoup plus exposés car l'environnement radiatif dans lequel évoluent les satellites est très différent de celui des orbites très basses des vols habités $(250 \mathrm{~km})$. Parmi les effets cumulatifs observés, citons l'effet de la dose cumulée qui entraîne la dérive des tensions de seuil des transistors MOS et dégrade le gain en courant des transistors bipolaires. L'emploi toujours croissant de composants commerciaux dans les systèmes embarqués et l'utilisation d'orbites basses liées aux exigences de la téléphonie moderne implique une parfaite connaissance de l'environnement. La dosimétrie embarquée a pour objectif de quantifier les contraintes radiatives auxquelles sont soumis les composants électroniques dans l'espace. La consommation et l'encombrement des lecteurs associés prohibent la lecture en vol des TLD. Les faibles débits de dose et surtout les variations brutales de l'environnement dues aux éruptions solaires ne permettent pas l'utilisation de tous les types de dosimètres. L'OSL est proposée comme une solution complémentaire aux dispositifs utilisés. La dynamique et la sensibilité de l'OSL permettent de répondre au souhait formulé par le CNES, à savoir de mesurer une variation de la dose orbite par orbite, quelles que soient les variations de l'environnement.

\section{Les principales propriétés des matériaux OSL utilisés : sources de stimulation et détecteurs}

(Lapraz et al., 2006 ; Dusseau, 1995 ; Beteille, 1995 ; Missous, 1992 ; Loup, 1991)

C'est le mode de stimulation du matériau qui caractérise les phénomènes de luminescence par OSL par rapport à l'ensemble des phénomènes de luminescence. Comme la plupart des matériaux luminescents, les matériaux OSL sont des isolants qui contiennent des impuretés dont les niveaux d'énergie sont situés dans la bande interdite. L'énergie apportée au matériau dopé lors de l'excitation, ou de l'irradiation, provoquera le piégeage des électrons et des trous sur les niveaux pièges associés à ces impuretés. Nous savons que la durée de vie des électrons et des trous sur ces niveaux dépend de leur profondeur et de la température. Dans le cas des matériaux OSL, le processus de stimulation (ou d'activation), qui provoque la libération des électrons et des trous piégés est un processus d'absorption optique (visible ou infra-rouge). Il convient de noter qu'un même matériau peut être à la fois thermoluminescent et stimulable optiquement. 


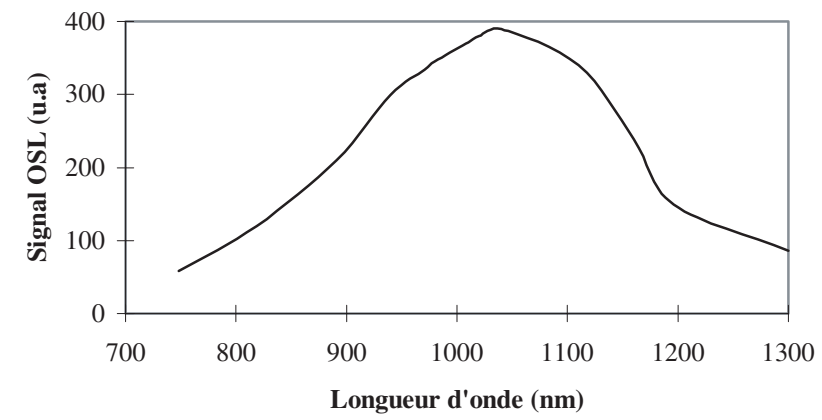

Figure 1 - Spectre de stimulation d'un échantillon de CaS:Ce,Sm (Beteille, 1995).

Stimulation spectra of CaS:Ce,Sm sample.

Quelle que soit l'application envisagée, c'est le matériau OSL qui constitue la clé de voûte du système. C'est donc lui qu'il convient de choisir en premier lieu, ce choix conditionnant par la suite les autres éléments du système. Les matériaux utilisés au laboratoire appartiennent à la famille de Lenard $(\mathrm{MgS}, \mathrm{CaS}, \mathrm{SrS}$, et $\mathrm{BaS}$ ). Afin d'optimiser la stimulation et la détection, nous devrons nous intéresser en premier lieu aux caractéristiques spectrales de nos matériaux.

\subsection{Les caractéristiques spectrales des sulfures alcalino-terreux}

On sait qu'en règle générale, les spectres de stimulation et d'émission des matériaux luminescents sont liés à la distribution des niveaux d'énergie dans la bande interdite du matériau hôte. Dans le cas des sulfures alcalino-terreux, les spectres de stimulation et d'émission sont pratiquement identiques quelle que soit la matrice hôte.

Le relevé du spectre de stimulation s'effectue en irradiant le matériau OSL aux UV (254 nm) puis en le stimulant avec différentes longueurs d'ondes comprises entre 700 et $1300 \mathrm{~nm}$. À titre d'exemple, nous donnons, figure 1, le spectre de stimulation du sulfure de calcium dopé cérium-samarium. Ce spectre, caractéristique du co-activateur (dans ce cas l'ion samarium), se situe dans l'infrarouge, il s'étend de 700 à $1300 \mathrm{~nm}$, avec un maximum autour de $1050 \mathrm{~nm}$.

Les courbes des figures 2 et 3 représentent les spectres d'émission OSL (Fig. 2) et d'excitation (Fig. 3) du sulfure de strontium dopé cérium-samarium. Ces spectres sont caractéristiques de l'ion $\mathrm{Ce}^{3+}$ au sein de la matrice $\mathrm{SrS}$ (ion activateur) (Lapraz et al., 2006).

Il est important de noter que les spectres sont indépendants du type de rayonnement d'excitation (photons UV, X, $\gamma$, électrons, etc.) et que du fait que les 


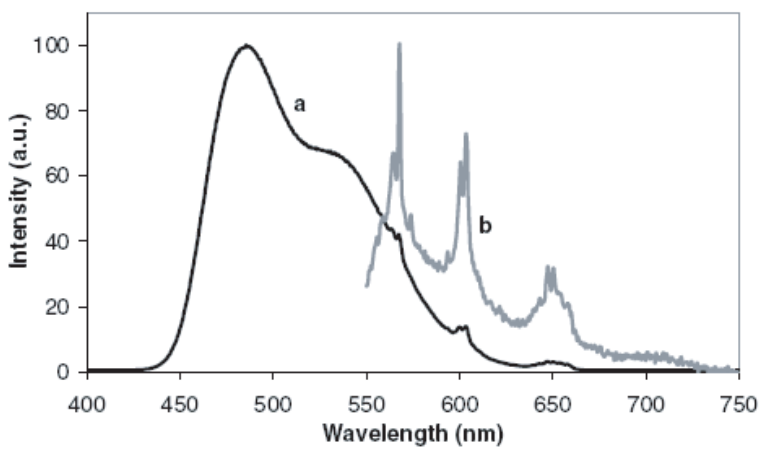

Figure 2 - Spectre d'émission du SrS:Ce,Sm avec $\lambda_{\text {excitation }}$ : (a) $292 \mathrm{~nm}\left(\mathrm{Ce}^{3+}\right)$, (b) $335 \mathrm{~nm}\left(\mathrm{Sm}^{3+}\right)$. Emission spectra of SrS:Ce,Sm.

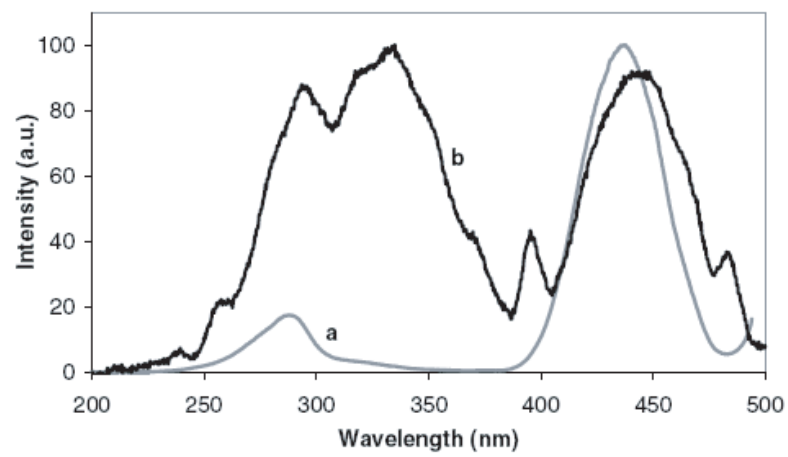

Figure 3 - Spectre OSL d'excitation du SrS:Ce,Sm, avec $\lambda_{\text {émission }}$ : (a) $504 \mathrm{~nm}\left(\mathrm{Ce}^{3+}\right),\left(\right.$ b) $650 \mathrm{~nm}\left(\mathrm{Sm}^{3+}\right)$. OSL excitation spectra of SrS:Ce,Sm.

spectres de stimulation et d'émission de ces matériaux, identiquement dopés, soient nettement séparés, il n'y aura pas de problèmes de détection de la lumière émise lors de la stimulation.

\subsection{La sensibilité et la dynamique des sulfures alcalino-terreux}

Au niveau de la sensibilité, les performances d'un capteur OSL seront directement liées aux caractéristiques du système de détection de la luminescence. Nous avons pu expérimentalement montrer qu'il était possible de détecter une dose minimum de $10 \mu \mathrm{Gy}$ après irradiation du matériau aux RX de $50 \mathrm{kV}$. 
Quant à la dynamique des matériaux, les travaux réalisés au Centre européen pour la recherche nucléaire (CERN) ont mis en évidence une limite de saturation de l'ordre de 300 Gy. Les études précédentes réalisées par le LETI sur nos matériaux en vue du développement d'un système OSL par fibre optique n'avaient pas montré de saturation jusqu'à 100 Gy (Dusseau et al., 1995).

En résumé, les caractéristiques intrinsèques des matériaux OSL sont les suivantes :

- des spectres de stimulation et d'émission bien séparés (Figs. 1-3);

- une grande dynamique ( $\geq 7$ ordres de grandeur) ;

- une bonne sensibilité $(\leq 10 \mu \mathrm{Gy})$;

- une réponse uniquement fonction de la dose absorbée ;

- un vidage complet du matériau après lecture (RAZ) ;

- un temps de lecture court, qui dépend de la puissance de stimulation et de la dose ;

- une sensibilité à tous les types de rayonnements ionisants.

\subsection{Les sources de stimulation et les dispositifs de détection}

Les sources de stimulation doivent se rapprocher au mieux du spectre de stimulation du matériau OSL. Ainsi, une lampe halogène filtrée peut être accordée sur le maximum du spectre. Dans ce cas, seule une infime partie du spectre de la lampe est utilisée. Une diode laser émettant à $1,3 \mu \mathrm{m}$ est légèrement décalée par rapport au maximum du spectre de stimulation. Néanmoins, ce moyen de stimulation peut être tout à fait adapté si sa puissance compense ce décalage. On peut également noter que l'augmentation de la puissance permet de diminuer le temps de dépiégeage mais augmente notablement le bruit (détection infrarouge par le photodétecteur) (Albert, 1997).

Les moyens de détection doivent coïncider avec le spectre d'émission visible du matériau OSL tout en étant le moins sensibles possible au rayonnement infrarouge de stimulation. Les caméras CCD sont très sensibles mais nécessitent l'utilisation de filtres du fait de leur forte sensibilité à l'infrarouge. Quant aux photomultiplicateurs (PM), ils couvrent très bien le rayonnement visible. Enfin, pour des applications où une sensibilité de l'ordre du mGy est suffisante, les photodiodes permettent de réduire les dimensions d'un système OSL afin de l'intégrer sur moins d'un $\mathrm{cm}^{2}$. Le tableau I résume quelques couples « détecteur source de stimulation » développés dans le cadre de différentes applications.

\section{La cartographie de dose OSL et ses applications}

(Idri, 2004 ; Polge, 2001 ; Ranchoux, 2001 ; Albert, 1997) 


\section{TABLEAU I}

Quelques exemples de couples détecteur - source de stimulation dans le cadre de différentes applications, ainsi qu'une illustration des résultats obtenus.

A few examples of detectors - stimulation source used for different applications with a picture of the results obtained.

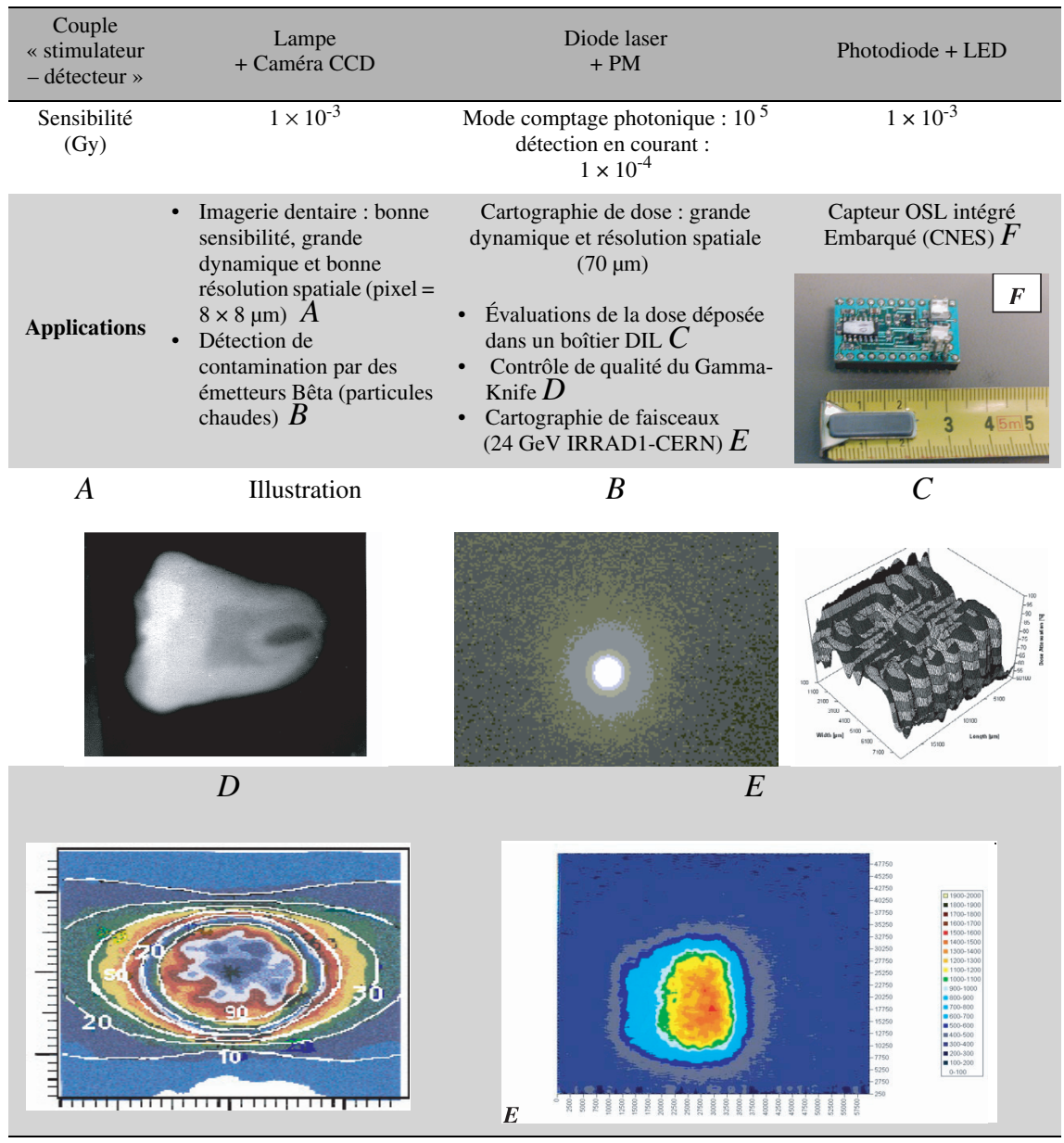

Le film OSL est obtenu par dépôt sérigraphié de matériau OSL sur un support en kapton (ou en polyéthylène). En fonction de l'application envisagée, l'épaisseur de matériau déposée varie de $10 \mu \mathrm{m}$ (pour des applications forte dose CERN kGy, radiothérapie Gy) à $100 \mu \mathrm{m}$ pour une sensibilité de l'ordre de $100 \mu \mathrm{Gy}$. Si dans son principe, la cartographie OSL est relativement simple, de nombreux paramètres 


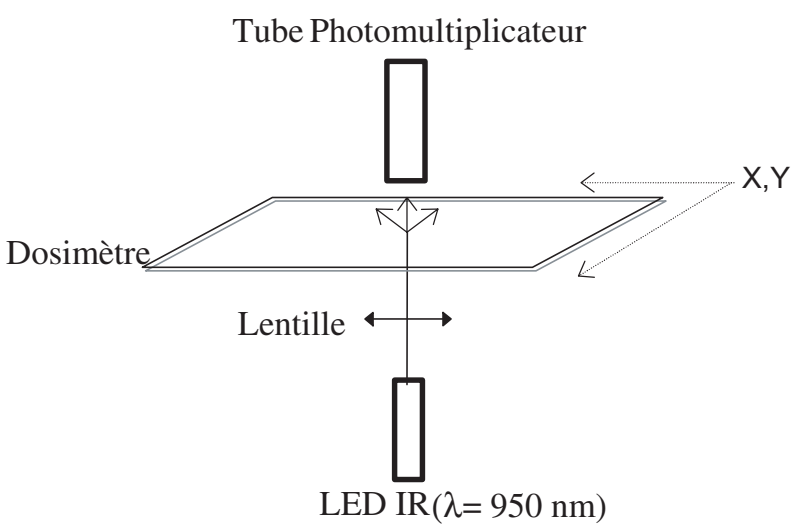

Figure 4. Principe du banc de lecture OSL.

$O S L$ readout system principle.

rendent complexe la mise au point d'un tel système. Le principe simplifié du banc de cartographie OSL est décrit sur la figure 4.

Une LED IR, dont la longueur d'onde d'émission du pic est centrée à $950 \mathrm{~nm}$, est focalisée dans le plan du détecteur. Le dosimètre est posé sur le plateau d'une table à déplacement X-Y permettant d'obtenir une stimulation continue par le laser selon les deux axes. Le film OSL se déplace donc à vitesse constante selon deux axes entre la source de stimulation et le PM qui restent fixes.

Chaque point stimulé émet un rayonnement visible proportionnel à la dose reçue et collecté au moyen du tube photomultiplicateur qui génère un courant proportionnel au nombre de photons collectés par sa photocathode. Il est ainsi possible d'obtenir, après un traitement et un étalonnage approprié de ce signal, des cartographies de doses en reliant chaque point stimulé à l'intensité du signal détecté.

À titre d'illustration, nous comparons dans le tableau II les caractéristiques des systèmes de cartographie TL et OSL mis au point au laboratoire et utilisés pour des applications dans les domaines de la radiothérapie, de l'espace et des faisceaux de particules de haute énergie.

En radiothérapie, le contrôle de qualité des installations et faisceaux revêt une importance toute particulière car tout dépassement de la dose délivrée au patient, ou toute erreur dans son positionnement, peut s'avérer critique. On sait, en effet, que la technique utilisée en oncologie consiste à irradier la zone tumorale au moyen de sources radioactives ou de faisceaux de particules issus d'accélérateurs. 
TABLEAU II

Comparaison des caractéristiques des systèmes OSL et TL.

Comparison between characteristics of OSL and TL readout.

\begin{tabular}{|c|c|c|}
\hline & $\mathrm{TL}$ & OSL \\
\hline STIMULATION & $\begin{array}{l}\text { Chauffage } \\
\text { laser } \mathrm{CO}_{2}\end{array}$ & LED IR $(950 \mu \mathrm{m})$ \\
\hline VOLUME & volume trop important de la manipulation & volume réduit \\
\hline BALAYAGE & point par point & continu (ligne par ligne) \\
\hline DÉTECTION & par intégrale du signal en chaque point & par détection de crête (forme du signal OSL) \\
\hline $\begin{array}{l}\text { TAILLE DES } \\
\text { CARTOGRAPHIES }\end{array}$ & $20 \times 20 \mathrm{~cm}^{2}$ & jusqu'à $20 \times 20 \mathrm{~cm}^{2}$ pour l'instant \\
\hline RÉSOLUTION & $1 \mathrm{~mm}$ & $50 \mu \mathrm{m}$ \\
\hline DYNAMIQUE & 7 décades & $\begin{array}{l}7 \text { décades } \\
\text { sensible à une large gamme de } \\
\text { rayonnements }\end{array}$ \\
\hline SENSIBILITÉ & $100 \mu \mathrm{Gy}$ & $20 \mu \mathrm{Gy}(\mathrm{X}), 100 \mu \mathrm{Gy}\left(\mathrm{e}^{-}\right)$ \\
\hline \multirow[t]{2}{*}{ PROBLÈME } & refroidissement du laser & pas de problème de refroidissement \\
\hline & \multicolumn{2}{|c|}{ DOSIMÈTRES ASSOCIÉS } \\
\hline MATÉRIAU & $\mathrm{CaSO}_{4}, \mathrm{Al}_{2} \mathrm{O}_{3}$ & $\mathrm{BaS}, \mathrm{CaS}, \mathrm{SrS}, \mathrm{MgS}$ \\
\hline SUPPORT & Kapton & kapton ou polyéthylène \\
\hline PRÉPARATION & Sérigraphie & sérigraphie \\
\hline
\end{tabular}

L'objectif des médecins est d'obtenir une dose absorbée très précise au niveau de la tumeur en évitant, autant que possible, d'exposer les tissus sains. La dose est déterminée par le praticien et des codes de calculs permettent alors de déterminer le temps d'irradiation nécessaire. La cartographie de dose apparaît comme une solution élégante pour permettre au praticien de visualiser la distribution spatiale de la dose absorbée et, de ce fait de contrôler ses calculs (Idri et al., 2004, 2001 ; Polge et al., 2001, 2000, 1999 ; Matias et al., 2000 ; Allières et al., 1998 ; Missous et al., 1995).

Dans le domaine de l'électronique spatiale, l'utilisation de composants électroniques durcis et les marges de sécurité importantes ont longtemps permis de s'affranchir des problèmes liés aux effets de dose. Toutefois, la nécessité de diminuer les coûts a cependant contraint les équipementiers à utiliser des composants commerciaux (COTS, commercial off the shelf) tout en réduisant au maximum les marges de sécurité. Dans ce contexte, l'évaluation exacte de la dose reçue par une puce de silicium à l'intérieur de son boîtier et dans l'environnement complexe de la plate-forme du satellite constitue un gage de fiabilité (Dusseau et al., 2000, 1999, 1998). Les codes de calculs ne peuvent prendre en compte tous les paramètres de la géométrie et limitent les cascades de particules secondaires 
TABLEAU III

Caractéristiques de l'environnement radiatif de diverses orbites (données CNES). Characteristics of the radiative environment for different orbit (CNES data).

\begin{tabular}{|c|c|c|c|c|}
\hline Orbite & $\begin{array}{l}\text { Caractéristiques } \\
\text { spatiales }\end{array}$ & Type de rayonnements & $\begin{array}{l}\text { Débit de dose } \mathrm{e}^{-} \\
(\mathrm{mGy} / \mathrm{mn}) \\
\text { derrière } 3 \mathrm{~mm} \text { d'Al }\end{array}$ & 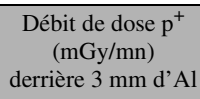 \\
\hline Polaire & $\begin{array}{c}800 \mathrm{~km} \\
98,8^{\circ}\end{array}$ & $\begin{array}{c}\text { Electrons piégés (ceinture } \\
\text { interne + SAA) } \\
\text { Protons piégés (ceinture + }\end{array}$ & $\begin{array}{c}\mathrm{D}_{\text {moy }}=0,025 \\
\mathrm{D}_{\max }=0,2\end{array}$ & $\begin{array}{c}\mathrm{D}_{\text {moy }}=0,005 \\
\mathrm{D}_{\max }=0,3\end{array}$ \\
\hline Jason & $\begin{array}{c}1336 \mathrm{~km} \\
60^{\circ}\end{array}$ & $\begin{array}{c}\text { Electrons piégés } \\
\text { Protons piégés + éruptions } \\
\text { solaires }\end{array}$ & $\begin{array}{c}\mathrm{D}_{\operatorname{moy}}=0,1 \\
\mathrm{D}_{\max }=2\end{array}$ & $\begin{array}{c}\mathrm{D}_{\operatorname{moy}}=0,1 \\
\mathrm{D}_{\max }=2\end{array}$ \\
\hline Géostationnaire & $\begin{array}{c}36000 \mathrm{~km} \\
0^{\circ}\end{array}$ & $\begin{array}{l}\text { Electrons piégés } \\
\text { Protons éruptions solaires }\end{array}$ & $\mathrm{D}_{\text {moy }}=0,8$ & $\mathrm{D}_{\max }=12,5$ \\
\hline GTO & $\begin{array}{c}360 \text { à } 36000 \mathrm{~km} \\
0^{\circ}\end{array}$ & $\begin{array}{l}\text { Electrons piégés } \\
\text { Protons piégés + éruptions } \\
\text { solaires }\end{array}$ & $\begin{array}{l}\mathrm{D}_{\text {moy }}=3,5 \\
\mathrm{D}_{\max }=10\end{array}$ & $\begin{array}{c}\mathrm{D}_{\text {moy }}=0,5 \\
\mathrm{D}_{\max }=8\end{array}$ \\
\hline
\end{tabular}

pour des questions de temps CPU. Dans ces conditions, la cartographie de dose OSL haute résolution s'impose comme un puissant moyen de contrôler et de calibrer les codes des calculs. C'est sur cette base que le CNES a financé un large programme de recherche au laboratoire sur les applications de l'OSL dans le domaine spatial (Ranchoux et al., 2000, 1999).

Le CERN dispose de grands accélérateurs de particules, dont les plus connus sont le Large Electron Positron (LEP) et le Large Hadron Collider (LHC) en construction. D'autres faisceaux comme IRRAD1 et T11 servent de moyens d'essai et d'irradiation. Les expériences CMS et Alice ont financé une campagne d'évaluation des possibilités offertes par l'OSL pour la caractérisation de ces faisceaux de particules de très hautes énergies. Sous forme de films, l'OSL a permis de réaliser la cartographie de faisceaux comme celui de protons de $24 \mathrm{GeV}$ délivré par IRRAD1 (Dusseau et al., 2001). La faisabilité d'un système de dosimétrie en ligne a aussi été démontrée (Dusseau et al., 2001 ; Dusseau, 2000).

\section{La dosimétrie embarquée}

Ravotti et al., 2004 ; Vaillé et al., 2005, 2003 ; Vaillé, 2003 ; Plattard et al., 2002 ; Dusseau et al., 2000)

Les premières missions expérimentales telles que le satellite MPTB de la NASA, ou le module ICARE du CNES, spécialement dédiées à l'étude de l'environnement radiatif, ont mis en évidence le caractère dynamique de l'environnement radiatif spatial (Tab. III). Le lancement du programme international Living With a Star a confirmé l'importance de cette étude. 
La durée de vie d'un satellite étant d'une dizaine d'années, il est impossible de prévoir les variations de débit de dose induites, par exemple, par les éruptions solaires. À partir de ce constat, l'idée selon laquelle la dose reçue par un satellite ou un équipement embarqué devait être contrôlée a fait son chemin. Un tel système doit de plus pouvoir donner l'alerte en cas d'augmentation critique du débit de dose.

Les systèmes actuellement utilisés dans le domaine spatial sont de deux types. Les capteurs intégrateurs enregistrant la dérive de transistors PMOS qui permettent d'obtenir une valeur précise de la dose reçue à l'échelle de temps d'une mission, sans remise à zéro possible. Les systèmes de comptages particule par particule, qui sont extrêmement sensibles mais saturent à fort débit de dose. On notera que les dosimètres thermoluminescents classiques qui nécessitent une exploitation au sol ne peuvent être embarqués que sur les vols habités.

L'expérience acquise au laboratoire en matière de dosimétrie utilisant des matériaux stimulables optiquement a permis de mettre au point un système complet (capteur + électronique intégrée) de faibles dimensions et de faible consommation électrique destiné à être embarqué sur un satellite et permettant de faire plusieurs mesures de dose à chaque orbite. La sensibilité de ce dosimètre intégrateur, de l'ordre de la dizaine de mGy, permet d'enregistrer la dose reçue orbite par orbite, avec remise à zéro à distance. On notera que la réalisation d'un tel dosimètre a nécessité une série d'études spécifiques, telles que l'analyse détaillée de l'environnement dans lequel doit fonctionner le dosimètre (température, débit de dose, dose cumulée, etc.) et surtout le durcissement de ce dispositif vis-àvis de son environnement. Par exemple, du fait de la sensibilité de la LED de stimulation aux effets des électrons, il a été nécessaire de mettre en place une boucle de contre-réaction permettant d'asservir la puissance optique de stimulation indépendamment du rendement de la LED. Grâce à ce durcissement au niveau circuit, pour des doses de l'ordre de $400 \mathrm{~Gy}$ ( $40 \mathrm{krad}$ ), soit une fluence d'électrons de l'ordre de $1 \times 10^{12} \mathrm{~cm}^{-2}$, aucune dégradation significative $(<5 \%)$ n'a été observée. Cette dégradation est de l'ordre de $30 \%$ en l'absence de durcissement.

Concernant la consommation électrique du capteur (paramètre très important pour l'installation à bord d'engins spatiaux), nous avons obtenu une consommation moyenne de l'ordre de $20 \mathrm{~mW}$, puissance qui est compatible avec les applications visées.

\section{Conclusion et perspectives}

Cet article dresse un état de l'art sur l'utilisation de matériaux OSL développés à l'IES pour la dosimétrie des rayonnements. Par le biais de nombreuses 
collaborations, tant au niveau national qu'international, un large spectre d'applications a vu le jour. Parmi celles-ci, nous citerons le contrôle géométrique de faisceaux avec les films OSL permettant l'étude de la distribution spatiale de la dose. L'utilisation de capteur intégré permet la réalisation de mesures de dose en ligne, cumulées ou fractionnées (avec remise à zéro). Ainsi, différents types de rayonnements (protons, électrons, photons et alpha) on été étudiés par ces dispositifs.

Nous souhaitons utiliser les dosimètres OSL pour la dosimétrie des neutrons. C'est la raison pour laquelle nous avons dopé nos matériaux au bore. Nous pourrons ainsi travailler dans le domaine des champs mixtes de radiations neutron - gamma tels que ceux rencontrés dans les réacteurs nucléaires ou les accélérateurs permettant la production de neutrons. Ainsi, des applications en thérapie par capture des neutrons par le bore (BNCT) ou en contrôle en environnement hostile pourraient voir le jour (thèse de doctorat de $\mathrm{D}$. Benoit en cours). Une étape de caractérisation a été menée afin d'étudier d'éventuelles modifications des propriétés, induites par le dopage au bore, de notre matériau. Aucune modification significative, excepté une baisse de luminescence dans le cas des échantillons borés, n'a été observée. Ce phénomène a été observé par Ravotti et al. dans le cadre d'une étude réalisée au CERN (Ravotti et al., 2005). Cette baisse de luminescence pourrait être attribuée à une relaxation sous forme de phonons plus importante par rapport au matériau initial. Il reste à vérifier cette hypothèse expérimentalement. Il serait nécessaire de mieux comprendre la distribution des dépôts d'énergie dans le cas du matériau boré et par conséquent d'étudier la répartition des atomes de bore dans la matrice. La réalisation du dopage au bore pourrait être modifiée en utilisant une opération par implantation ionique. Par ailleurs, des simulations type Monte Carlo pourraient être réalisées afin de mieux comprendre la réponse de nos matériaux borés. Parallèlement à cet axe, et afin d'améliorer la reproductibilité des dépôts OSL, une méthodologie par couches minces est en cours de réalisation (thèse de doctorat de P. Garcia en cours).

\section{RÉFÉRENCES}

Albert L. (1997) Cartographie de dose à partir de capteurs utilisant des matériaux phosphorescents, Thèse de l'université Montpellier II.

Albert L., Defez D., Dusseau L., Fesquet J., Gasiot J., Fau P., Gassa F., Porcheron D. (1997) Dose mapping system based on laser heating thermoluminescence plates. Discussion and comparison with results about gamma knife. In : World Congress on Medical Physics and Biomedical Engineering, September 14-19, Nice, France.

Allières N., Delard R., Dubois J.B., Dusseau L., Salomon O., Fesquet J. (1998) Dose mapping system based on optical stimulation Application to IORT. In : First International Congress of the society of IOR, Pamplona, Espagne.

Beteille D. (1995) Étude des potentialités des matériaux luminescents stimulables optiquement en vue d'applications en autoradiographie et en imagerie bêta, Thèse de l'université Montpellier II. 
Beteille D., Setzkorn R., Prévost H., Dusseau L., Fesquet J., Gasiot J. (1996) Laser Heating of thermoluminescentce plate: application to intraoperative radiotherapie, Med. Phys. 23, 1- 4.

Dusseau L., Bestion N., Fesquet J., Gasiot J. (1995) Faisabilité d'un lecteur portable de dosimètres OSL. Dans : Conférence des Laboratoires Associés pour la Recherche en Dosimétrie, CEA Saclay, France.

Dusseau L. (1995) Étude des potentialités des phénomènes de luminescence par stimulation optique (OSL) dans les sulfures alcalino-terreux. Applications à la réalisation d'un lecteur portable de dosimètres OSL, Thèse de l'université Montpellier II.

Dusseau L., Polge G., Albert L., Magnac Y., Bessiere J.C., Fesquet J., Gasiot J. (1998) Irradiated Integrated Circuits dose attenuation mapping using optically stimulated phosphors for packaging dosimetry, IEEE Trans. Nucl. Sci. 82, 2695-2699.

Dusseau L., Ranchoux G., Polge G., Plattard D., Magnac Y., Saigné F., Bessière J.C., Fesquet J., Gasiot J. (1999) High Energy Electrons Dose Mapping Using Optically Stimulated Luminescent Films, IEEE Trans. Nucl. Sci. 46, 1757-1761.

Dusseau L., Plattard D., Polge G., Ranchoux G., Saigné F., Fesquet J., Gasiot J. (2000) An Integrated sensor Using Optically Stimulated Luminescence For In Flight Dosimetry, IEEE Trans. Nucl. Sci. 47, 2412-2416.

Dusseau L. (2000) Optically Stimulated Luminescence Dosimetry. In : First CMS Workshop on Radiation Monitoring, CERN Genève, Suisse.

Dusseau L., Polge G., Mathias S., Vaillé J.-R., Germanicus R., Broadhead R., Camanzi B., Glaser M., Saigné F., Fesquet J., Gasiot J. (2001) High Energy Particles Irradiation of Optically Stimulated Luminescent Films at CERN, IEEE Trans. Nucl. Sci. 48, 2056-2060.

Gasiot J., Braunlich P., Fillard J.P. (1981) Laser heating in thermoluminescent dosimetry, J. Appl. Phys. 53, 5200-5209.

Gasiot J., Braunlich P., Fillard J.P. (1982) Nanosecond infared laser stimulation of luminescence rareearth doped AES, Appl. Phys. Lett. 40, 376-379.

Idri K., Polge G., Dusseau L., Fesquet J., Gasiot J., Dubois J.B., Delard R., Aillières N. (2001) Application de la cartographie de dose par luminescence optiquement stimulée à la radiothérapie per-opératoire. Dans : $40^{e}$ Congrès de la Société Française des Physiciens Médicaux, June 6-8, Nantes, France.

Idri K. (2004) Développement d'un système de cartographie par luminescence stimulée optiquement. Applications aux contrôles des traitements en radiothérapie avec modulation d'intensité et aux contrôles des faisceaux de protons de haute énergie, Thèse de l'université Toulouse III - Paul Sabatier.

Idri K., Santoro L., Charpiot E., Herault J., Costa A., Aillères N., Delard R., Vaillé J.-R., Fesquet J., Dusseau L. (2004) Quality control of Intensity Modulated Radiation Therapy with Optically Stimulated Luminescent films, IEEE Trans. Nucl. Sci. 51, 3638-3641.

Lapraz D., Prévost H., Idri K., Angellier G., Dusseau L. (2006) On the PL, TSL and OSL properties of SrS:Ce,Sm phosphor, Phys. Status Solidi A. 203, 3793-3800.

Loup F. (1991) Contribution à l'étude de la luminescence par stimulation optique dans les sulfures alcalino-terreux, utilisation du sulfure de magnésium en dosimétrie des radiations ionisantes, Thèse de l'université Montpellier II.

Matias S., Polge G., Plattard D., Dusseau L., Ranchoux G., Iborra-Brasart N., Hérault J. (2000) Contrôle d'un cyclotron médical par cartographie de dose en OSL. Dans : Congrès de la Société Française des Physiciens Médicaux, June 13-16, Lille, France.

Missous O. (1992) Contribution à l'étude de la luminescence par stimulation optique dans le sulfure de magnésium dopé cérium - samarium en vue de son utilisation en dosimétrie, Thèse de l'université Montpellier II. 
Missous O., Dusseau L., Fesquet J., Gasiot J. (1995) Applications de la luminescence stimulée optiquement et thermiquement à la détection du rayonnement. Dans : Colloque Instrumentation en Biologie et en Médecine, Paris, France, (conférence invitée), Académie des Sciences.

Plattard D. (2001) Étude de la faisabilité d'un capteur basé sur la stimulation optique (OSL), Thèse de l'université Montpellier II.

Plattard D., Ranchoux G., Dusseau L., Polge G., Vaille J.-R., Gasiot J., Fesquet J., Ecoffet R., IborraBrassart N. (2002) Characterization of an integrated sensor using optically stimulated luminescence for in-flight dosimetry, IEEE Trans. Nucl. Sci. 49 1322-1326.

Polge G. (2001) Étude de la faisabilité d'un système de cartographie de dose par stimulation optique et application à la radiothérapie à haut gradient de dose, Thèse de l'université Montpellier II.

Polge G., Dusseau L., Gensanne D., Plattard D., Saigné F., Bessiere J.C., Fesquet J., Gasiot J., Allières N., Delard R., Dubois J.B. (1999) Application de la cartographie de dose par luminescence stimulable optiquement au contrôle de la qualité du gamma knife. Dans : Congrès de la Société Française des Physiciens d'Hôpitaux, May 19-21, Tours, France.

Polge G., Dusseau L., Matias S., Gensanne D., Plattard D., Fesquet J., Gasiot J., McNulty P.J., Davis M., Tortora M., Porcheron D., Dubois J.B., Aillières N., Delard R. (2000) Applications to Radiotherapy of a Dose-Mapping System Based on the Optically Stimulated Luminescence. Dans : IEEE Medical Imaging Conference, Lyon, France.

Polge G., Dusseau L., Plattard D., Fesquet J., Gasiot J., Iborra-Brassard N. (2001) Characterization of a $63 \mathrm{MeV}$ Proton Beam with Optically Stimulated Luminescent Films. Dans : $\sigma^{e}$ Conférence Européenne RADECS, September 10-14, Grenoble, France.

Prévost H., Setzkorn R., Beteille D., Dusseau L., Albert L., Fesquet J., Gasiot J. (1995) Technique de thermoluminescence par chauffage laser appliquée à l'imagerie des sources bêta. Dans : Conférence des Laboratoires Associés pour la Recherche en Dosimétrie, CEA Saclay.

Ranchoux G., Dusseau L., Gensanne D., Plattard D., Polge G., Saigné F., Bessiere J.C., Fesquet J., Gasiot J. (1999) Rendement en profondeur dans un boîtier de composants électroniques au moyen de films luminescents stimulables optiquement. Dans : $5^{e}$ Conférence Européenne RADECS, September 13-17, Fontevraud, France.

Ranchoux G., Plattard D., Polge G., Dusseau L., Fesquet J., Gasiot J., Ecoffet R., Iborra-Brassart N. (2000) Proton Dosimetry Measurements and Calculations In Electronic Packages Using Optically Stimulated Luminescent Film. Dans : Workshop RADECS, September 11-13, Louvain La Neuve, Belgique.

Ranchoux G. (2001) Étude et réalisation de détecteurs utilisant des matériaux phosphorescents stimulables optiquement pour des applications en dosimétrie, Thèse de l'université Montpellier II.

Ravotti F., Glaser M., Moll M., Idri K., Vaillé J.-R., Prevost H., Dusseau L. (2004) Conception of an Integrated Sensor for the Radiation Monitoring of the CMS Experiment at the Large Hadron Collider, IEEE Trans. Nucl. Sci. 51, 3642-3648.

Ravotti F., Glaser M., Idri K., Vaillé J.-R., Prevost H., Dusseau L. (2005) Optically stimulated luminescence materials for wide-spectrum neutron measurements at CERN, IEEE Tans. Nucl. Sci. 52, 1568-1573.

Setzkorn R. (1995) Adaptation de la technique de thermoluminescence par chauffage laser à la dosimétrie des rayonnements bêta, Thèse de l'université Montpellier II.

Setzkorn R., Beteille D., Dusseau L., Prévost H., Fesquet J., Gasiot J. (1996) Dosimétrie des particules chaudes utilisant la thermoluminescence par chauffage laser et par stimulation optique, Radioprotection 31, 83-90.

Setzkorn R., Magnac Y., Prévost H., Nadai J.P., Albert L., Dusseau L., Fesquet J. (1997) Hot particles dosimetry using laser heating thermoluminecence foils compared to EGS4 PRESTA calculations, Rad. Prot. Dosim. 72, 119-126. 


\section{LA LUMINESCENCE STIMULÉE OPTIQUEMENT}

Sevière H. (1993) Cartographie de dose gamma en champ mixte neutron - gamma en milieu modérateur: mise au point d'une capteur thermoluminescent et d'un lecteur laser, Thèse de l'université Montpellier II.

Vaillé J.-R. (2003) Développement, durcissement et caractérisation d'un dosimètre OSL pour applications spatiales, Thèse de l'université Montpellier II.

Vaillé J.-R., Ducret S., Idri K., Saigne F., Matias S., Iborra-Brasart N., Germanicus R., Ecoffet R., Dusseau L. (2003) Hardening of a radiation sensor based on optically stimulated luminescence, IEEE Trans. Nucl. Sci. 50, 2358-2362.

Vaillé J.-R., Ravotti F., Garcia P., Glaser M., Matias S., Idri K., Boch J., Lorfèvre E., McNulty P.J., Saigné F., Dusseau L. (2005) Online dosimetry based on optically stimulated luminescent materials, IEEE Trans. Nucl. Sci. 52, 2578-2582.

Vignolo C. (1994) Application des techniques de lectures par chauffage laser de plaques thermoluminescentes pour l'autoradiographie, Thèse de l'université Montpellier II. 